\title{
Heterogeneity in polymer melts from melting of polymer crystals
}

SANJAY RASTOGI, DIRK R. LIPPITS, GERRIT W. M. PETERS, ROBERT GRAF, YEFENG YAO AND HANS W. SPIESS

Nature Materials 4, 635-641 (2005)

The accepted date given for this article was incorrect, it should have read 22 June 2005. 